\title{
Estudo da Estabilização Termo-0xidativa de Fitas e Feixes de Fibras de Poliacrilonitrila
}

\author{
Andréa M. A. R. Costa, Sergio P. Ravagnani \\ Departamento de Tecnologia de Polímeros, FEQ, UNICAMP
}

\author{
Antônio P. Del' Arco Jr. \\ Divisão de Materiais, IAE, CTA
}

Resumo: Os estudos relacionados ao processo de fabricação de fibras de carbono indicam que a etapa de estabilização termo-oxidativa das fibras precursoras é limitante, envolvendo reações químicas complexas e mudanças na estrutura física das fibras. Com o objetivo de se conhecer melhor a etapa de estabilização e eliminar gastos em testes experimentais, a modelagem e a simulação surgem para tentar otimizar este processo. Apresenta-se neste trabalho um modelo matemático para a etapa de estabilização de uma fita de fibras de poliacrilonitrila. As equações do modelo descrevem as principais reações químicas de estabilização, a difusão de oxigênio e a transferência de calor na fita. Para a resolução do modelo foi desenvolvido um programa computacional na linguagem Fortran, utilizando os métodos de Colocação Ortogonal e Runge- Kutta Semiimplícito. Foram obtidos resultados de temperaturas e concentrações na fita de fibras de poliacrilonitrila em função do tempo de estabilização e da posição na espessura da fita. Estes resultados foram analisados e comparados com os simulados em um feixe de fibras de poliacrilonitrila e indicaram que a transferência de calor na fita é melhor do que no feixe e conseqüentemente as reações exotérmicas são mais controladas.

Palavras-chave: Fibra de poliacrilonitrila, estabilização termo-oxidativa, modelo matemático.

\section{Study of Thermo-0xidative Stabilization for Strips and Bundles of Polyacrylonitrile Fibers}

\begin{abstract}
Stabilization is one of the expensive and critical steps in the manufacture of carbon fibers. It involves complex chemical reactions and physical structure changes. Modeling and simulation are successfully utilized in order to know the stabilization behavior and minimize the cost of experimental tests. In this work a mathematical model of stabilization step is presented for a polyacrylonitrile strip. Differential equations describe the chemical reactions, oxygen diffusion and heat transfer occurring inside the strip. They are resolved by Orthogonal Collocation and Semi implicit Runge - Kutta Method. The results of temperatures and concentrations profiles as a function of the stabilization time are analyzed and compared with a simulation obtained for a bundle of polyacrylonitrile fibers. This investigation indicates that heat transfer in the strip is better than in the bundle and consequently the exothermic reactions are controlled.
\end{abstract}

Keywords: Polyacrylonitrile fiber, thermo-oxidative stabilization, mathematical model.

\section{Introdução}

Comercialmente as fibras de carbono são produzidas pela decomposição térmica de fibras precursoras compostas de copolímeros de poliacrilonitrila (PAN). No processo de fabricação de fibras de carbono, a PAN precursora é primeiramente fiada e esti- rada para se obter uma orientação preferencial na molécula do polímero, seguindo-se a estabilização em ar e, para finalizar, a carbonização da fibra estabilizada em atmosfera inerte.

O longo tempo de processamento associado à importância da completa oxidação faz da estabilização um primeiro candidato à modelagem do processo. A

Autor para correspondência: Sergio P. Ravagnani, FEQ/UNICAMP, Caixa Postal:6066, CEP: 13081-970, Campinas, SP. E-mail:ravag@dtp.feq.unicamp.br 
modelagem da estabilização requer dois tipos de informações: as expressões cinéticas para as reações de ciclização, desidrogenação e oxidação e a informação sobre a difusão de oxigênio e calor através do polímero em reação. A maior limitação para a modelagem da estabilização se deve à insuficiência de dados cinéticos para as reações. Apesar desta limitação, os resultados obtidos de trabalhos de simulação foram satisfatórios.

Grove e Abhiraman (1992) desenvolveram um modelo simplificado para a estabilização em uma fibra sólida de PAN. Este modelo considera uma estabilização em batelada ocorrendo em meios inerte e oxidante, e só é válido para baixas temperaturas de estabilização, onde as reações são controladas e pode-se admitir condições isotérmicas através da fibra. $\mathrm{O}$ modelo acompanha a conversão de alguns grupos químicos, como: grupos nitrila, oxigênio livre e oxigênio reagido na fibra.

Dunham e Edie (1992) combinaram uma modelagem matemática com dados experimentais e obtiveram um modelo para o processo de estabilização. Este modelo considera um feixe homogêneo de fibras PAN sofrendo as principais reações de estabilização: ciclização, desidrogenação e oxidação. Incluíram ainda a difusão de oxigênio e a transferência de calor ocorrendo em grandes feixes de fibras PAN. Os dados experimentais indicaram que o modelo descreve bem as temperaturas e composições para feixes de 12000 a 60000 filamentos e que a temperatura do feixe pode ficar aproximadamente $15{ }^{\circ} \mathrm{C}$ acima da temperatura do forno.

Del' Arco (1997) obteve soluções matemáticas na determinação de oxigênio difundido e reagido na fibra PAN, após a estabilização oxidativa.

Recentemente, obtivemos resultados simulados de temperaturas e concentrações em um feixe de fibras PAN (Rosa (1999)), onde foi verificada a importância da temperatura do forno, da direção e velocidade do fluxo de ar no forno e do número de filamentos no feixe para controlar as reações exotérmicas de estabilização. O modelo matemático utilizado neste trabalho foi desenvolvido por Dunham e Edie (1992).

Como o processo industrial estabiliza fitas planas de fibras PAN, o objetivo agora é desenvolver um modelo matemático para este conjunto de fibras e o que basicamente mudará em relação ao modelo de feixe é a forma geométrica do conjunto de fibras.

Propomos então um modelo matemático para a etapa de estabilização de uma fita de fibras PAN. As equações deste modelo descrevem as principais rea- ções químicas de estabilização, a difusão de oxigênio e a transferência de calor na fita. O objetivo deste trabalho é o de determinar os perfis de temperaturas e concentrações na fita de fibras PAN, analisá-los e compará-los com os perfis simulados em um feixe de fibras PAN obtidos em nosso trabalho anterior (Rosa, 1999).

\section{Modelagem Matemática para a Estabilização de Fitas de Fibras PAN}

\section{Interpretação Física}

O modelo proposto para a estabilização de um feixe de fibras PAN (Dunham e Edie, 1992 e Rosa, 1999) considerou um conjunto de fibras PAN formando o feixe (forma cilíndrica), como apresentado na figura 1 .

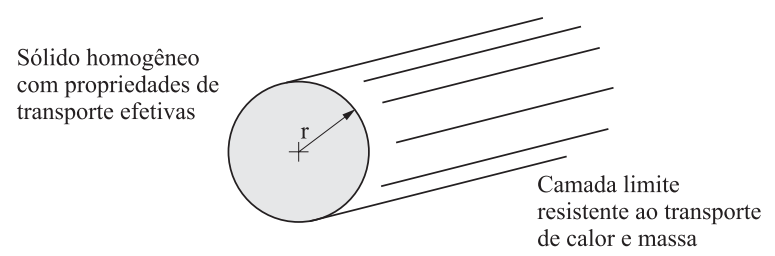

Figura 1. Diagrama do modelo de um feixe de fibras PAN (Dunham e Edie, 1992).

Para a disposição plana de um conjunto de fibras formando uma fita a interpretação é a seguinte:

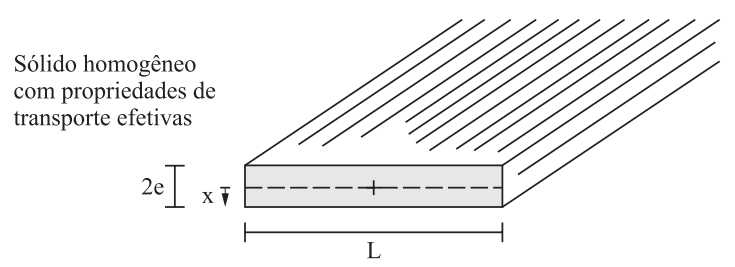

Figura 2. Diagrama do modelo de uma fita de fibras PAN.

O que muda em relação à modelagem obtida para o feixe é justamente a forma geométrica do conjunto de fibras e conseqüentemente a forma de difusão de oxigênio e transferência de calor nas fibras.

\section{Reações Químicas}

As equações do balanço de massa para as reações químicas são as mesmas propostas para o modelo de feixe (Dunham e Edie, 1992 e Rosa, 1999). Foram considerados três grupos representativos de reações: a ciclização do grupo nitrila;
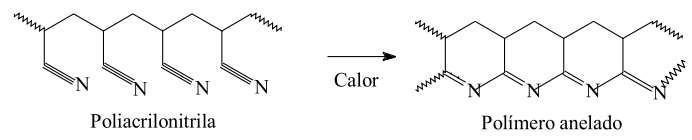
a desidrogenação da ligação carbono-carbono;

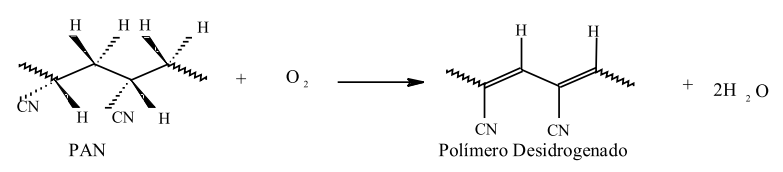

e a oxidação.

$$
P+\frac{1}{2} O_{2} \rightarrow P-O-R
$$

onde $\mathrm{P}$ representa um segmento de polímero não oxidado e P-O-R representa um segmento oxidado com um ligante geral $\mathrm{R}$.

\section{Balanço de Massa}

A partir de um balanço de massa para grupos nitrila, polímero desidrogenado e ligação oxigênio, obtêm-se equações em função do tempo e de forma adimensionalizada, $\mathrm{y}_{\mathrm{CN}}, \mathrm{y}_{\mathrm{C}=\mathrm{C}}, \mathrm{y}_{\mathrm{O}}$, tem-se:

$$
\begin{aligned}
& \frac{\partial y_{C N}}{\partial t}=-k_{1}\left(1-e^{-k_{i} t}\right) y_{C N} \\
& \frac{\partial y_{C=C}}{\partial t}=k_{2} C_{O_{2} x i} y_{O_{2}}\left(1-y_{C=C}\right) \\
& \frac{\partial y_{O}}{\partial t}=k_{3} C_{O_{2} x i} y_{O_{2}}\left(1-y_{O}+y_{O i}\right)
\end{aligned}
$$

As concentrações do grupo nitrila $\left(\mathrm{y}_{\mathrm{CN}}\right)$, da dupla ligação $\left(\mathrm{y}_{\mathrm{C}=\mathrm{C}}\right)$ e a concentração de ligação oxigênio $\left(\mathrm{y}_{\mathrm{O}}\right)$, foram adimensionalizadas pela concentração de unidade repetitiva $\left(\mathrm{C}_{\text {rep }}\right)$ e a concentração de oxigênio livre na fita $\left(\mathrm{y}_{\mathrm{O} 2}\right)$ foi normalizada pela concentração de oxigênio no ar do forno $\left(\mathrm{C}_{\mathrm{O} 2 \mathrm{xi}}\right)$. As derivadas são parciais, pois as concentrações dependem da posição na espessura (x) e do tempo (t). O balanço de massa com relação à nitrila apresenta um termo de atraso exponencial originado pela reação de iniciação, onde $\mathrm{k}_{\mathrm{i}}$ é a velocidade para iniciar a reação de ciclização (Collins, Thomas e Williams, 1988).

A solução destas três equações diferenciais parciais de primeira ordem requer somente as condições iniciais de concentrações $\left(\mathrm{y}_{\mathrm{CNi}}=1 ; \mathrm{y}_{\mathrm{C}=\mathrm{Ci}}=0\right.$ e $\mathrm{y}_{\mathrm{Oi}}=$ oxigênio presente nos comonômeros).

A difusão de oxigênio na estabilização de um feixe de fibras acontece radialmente, já a difusão em uma fita ocorre na espessura. De acordo com as leis de Fick, a concentração adimensionalizada de oxigênio livre $\mathrm{y}_{\mathrm{O} 2}$ é descrita por:

$$
\begin{aligned}
& \frac{\partial y_{O_{2}}}{\partial t}=\frac{D_{e}}{e^{2}} \frac{\partial^{2} y_{O 2}}{\partial x *^{2}}-k_{2} C_{r e p} y_{O_{2}}\left(1-y_{C=C}\right) \\
& -\frac{1}{2} k_{3} C_{r e p} y_{O_{2}}\left(1-y_{O}+y_{O i}\right)
\end{aligned}
$$

onde $\mathrm{x}$ * é a distância adimensionalizada da espessura da fita, $x^{*}=x$ / e. A solução desta equação requer uma condição inicial e duas condições de contorno.

Condição inicial: o oxigênio livre inicial é aproximadamente o oxigênio contido no ar entre as fibras.

Condição de contorno 1: é aplicada na metade da espessura da fita, ou seja a condição de simetria.

$$
\frac{\partial y_{O_{2}}}{\partial x^{*}}(0, t)=0
$$

Condição de contorno 2: é determinada na superfície da fita, onde a transferência de massa é descrita pela equação de difusão de oxigênio, com a velocidade de convecção de oxigênio através da camada limite da superfície.

$$
\frac{\partial y_{O_{2}}}{\partial x^{*}}(1, t)=\operatorname{Sh}\left(y_{O_{2} x}-y_{O_{2}}(1, t)\right)
$$

onde $\mathrm{y}_{\mathrm{O} 2 \mathrm{x}}$ (concentração de oxigênio no ar do forno) é dado por:

$$
y_{O_{2} x}=\frac{C_{O_{2} x}}{C_{O_{2} x i}}
$$

e Sh é o número de Sherwood, uma forma adimensionalizada do coeficiente de transferência de massa, $\mathrm{k}_{\mathrm{m}}$. As constantes de velocidade $\mathrm{k}, \mathrm{k}_{2}$ e $\mathrm{k}$ são obtidas pela equação de Arrhenius:

$$
k_{j}=k_{0, j} e^{-E_{j} / R T_{f}}
$$

onde $\mathrm{k}_{\mathrm{j}}$ é a constante de velocidade para a reação $\mathrm{j}$, $\mathrm{k}_{0, \mathrm{j}}$ é o fator de freqüência de Arrhenius para a reação $\mathrm{j}, \mathrm{E}_{\mathrm{j}}$ é a energia de ativação para a reação j e R é a constante universal dos gases.

\section{Transferência de Calor}

As fibras de PAN devem ser estabilizadas a temperaturas relativamente baixas para prevenir reações 
descontroladas. O balanço de energia considera a transferência de calor do forno para a fita em função da espessura e o calor gerado pelas reações químicas exotérmicas, ou seja:

$$
\begin{aligned}
\frac{\partial \theta}{\partial t}= & \frac{\alpha_{e}}{e^{2}} \frac{\partial^{2} \theta}{\partial(x *)^{2}}+\frac{\left(-\Delta H_{1}\right)}{T_{x i} \rho c_{p}} k_{1} C_{r e p}\left(1-e^{-k_{i} t}\right) y_{C N} \\
& +\frac{\left(-\Delta H_{2}\right)}{T_{x i} \rho c_{p}} k_{2} C_{r e p} C_{O_{2} x i} y_{O_{2}}\left(1-y_{C=C}\right) \\
& +\frac{\left(-\Delta H_{3}\right)}{T_{x i} \rho c_{p}} k_{3} C_{r e p} C_{O_{2} x i} y_{O_{2}}\left(1-y_{O}+y_{O i}\right)
\end{aligned}
$$

onde $\rho$ é a densidade da fita, $\mathrm{C}_{\mathrm{p}}$ é a capacidade calorífica da fita, $\mathrm{k}_{\mathrm{e}}$ é a condutividade térmica efetiva da fita homogênea. A pressão e a condutividade térmica efetiva são assumidas constantes. A temperatura é adimensionalizada da seguinte forma:

$$
\theta=\frac{T_{f}-T_{x}}{T_{x i}}
$$

onde $\mathrm{T}_{\mathrm{xi}}$ é a temperatura inicial do forno. A difusividade térmica efetiva, $\alpha_{\mathrm{e}}$ é análoga à difusividade efetiva no balanço de oxigênio.

Como no balanço de oxigênio, a solução desta equação diferencial requer uma condição inicial e duas condições de contorno.

Condição inicial: é a temperatura inicial da fita, $\mathrm{T}_{\mathrm{fi}}$

Condição de contorno 1: condição de simetria.

$$
\frac{\partial \theta}{\partial x *}(0, t)=0
$$

Condição de contorno 2: é determinada na superfície da fita.

$$
\frac{\partial \theta}{\partial x *}(1, t)=- \text { Biot } \theta(1, t)
$$

onde o Número de Biot pode ser interpretado como a razão entre resistência do fluxo de calor interno e a resistência do fluxo de calor externo, definido adimensionalmente por:

$$
\text { Biot }=\frac{h \cdot e}{k_{e}}
$$

$\mathrm{O}$ valor de $\mathrm{k}_{\mathrm{e}}$ refere-se ao material sólido e $\mathrm{h}$ é determinado pela correlação para o número de Nusselt.

\section{Resolução das Equações do Modelo}

As variáveis dependentes preditas pelo modelo são temperatura, $\theta$, e as concentrações das quatro espécies reativas, $\mathrm{y}_{\mathrm{CN}}, \mathrm{y}_{\mathrm{C}=\mathrm{C}}, \mathrm{y}_{\mathrm{O}}$ e $\mathrm{y}_{\mathrm{O} 2}$. As cinco equações que descrevem as variáveis dependentes e independentes contêm muitos parâmetros de transporte, propriedades físicas e constantes cinéticas de reações. Todos estes dados de constantes requeridos pelo modelo foram encontrados em pesquisas anteriores (Dunham e Edie, 1992 e Rosa, 1999).

As equações dos balanços de massa e energia dependem da espessura da fita $\left(\mathrm{x}^{*}\right)$ e do tempo de estabilização (t). Com a aplicação da colocação ortogonal obtêm-se equações diferenciais ordinárias que dependem somente do tempo de estabilização. A resolução de uma equação diferencial através do método de Colocação Ortogonal permite uma aproximação das derivadas da função por somatórios ponderados dos valores da função nos pontos de colocação. A derivada para um determinado ponto de colocação é expressa em termos da solução de todos os pontos de colocação. Uma explicação detalhada deste método pode ser encontrada nas publicações de Villadsen (1970), Villadsen e Michelsen (1978) e Finlayson (1980).

Neste trabalho, para a determinação dos pontos de colocação, foram utilizados os polinômios de Jacobi, conforme Villadsen e Michelsen (1978), que são ortogonais no intervalo $[0,1]$. Os pontos de colocação são tomados como as raízes do polinômio de Jacobi $\mathrm{P}_{\mathrm{N}}{ }^{(\mathrm{a}, \mathrm{b})}$ ou simplesmente $\mathrm{N}(\alpha, \beta)$, dado por:

$$
\int_{0}^{1} x^{\beta}(1-x)^{\alpha} x^{j} P_{N}^{(\alpha, \beta)}(x) d x=0 \quad \mathrm{j}=1,2, \ldots, \mathrm{N}
$$

Aplica-se então um método de integração e neste caso utilizou-se o Método de Runge-Kutta Semi-implícito (Michelsen, 1976), já que este método é utilizado com eficiência na resolução de equações diferenciais ordinárias com estabilidade superior a outros métodos de integração.

\section{Resultados e Discussão}

Foi desenvolvido um programa computacional na linguagem Fortran para resolver as equações do modelo. Com a resolução destas equações são obtidos os perfis de temperaturas e concentrações em uma fita de fibras PAN durante a estabilização. Através 
destes perfis pôde-se analisar o comportamento das reações exotérmicas de estabilização e verificar as diferenças que ocorrem entre uma estabilização em um feixe e em uma fita de fibras PAN.

\section{Perfis de temperaturas e concentrações em função} da espessura da fita

Na Figura 3 apresentam-se os perfis de temperatura em função de $\mathrm{x}^{*}$ em quatro tempos durante a estabilização, e na Figura 4 apresentam-se os perfis de concentrações em função de $\mathrm{x}^{*}$ após 2500 segundos de estabilização. Estas simulações foram feitas para fitas de 50000 filamentos, em um forno isotérmico a $240{ }^{\circ} \mathrm{C}$ e com velocidade do ar no forno de $300 \mathrm{~cm} / \mathrm{s}$.

Estes perfis quase constantes foram obtidos também para um feixe de fibras PAN e indicam que mesmo para grandes feixes ou fitas, a estabilização ocorre de forma uniforme no raio do feixe ou espessura da fita.

Para a simulação das concentrações e temperaturas em função do tempo foram utilizados 7 pontos de

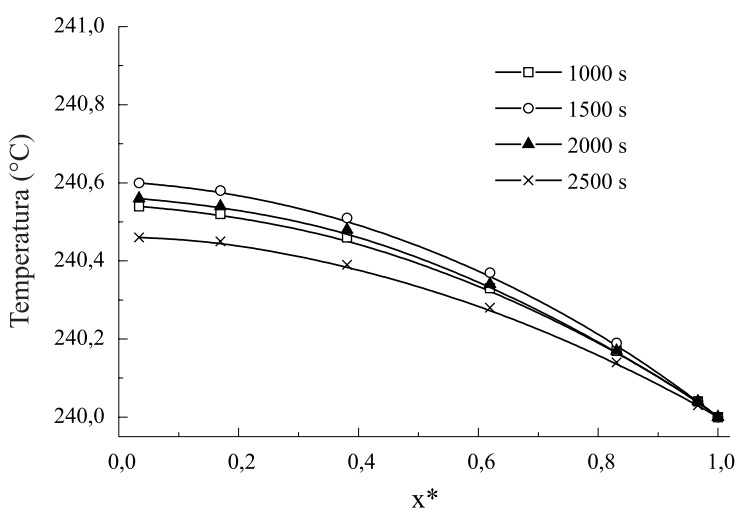

Figura 3. Perfis de temperatura na fita de fibras PAN em função de $x^{*}$ depois de 1000, 1500, 2000 e 2500 segundos de estabilização, para valores de $\mathrm{N}+1=7, \alpha=0$ e $\beta=0$.

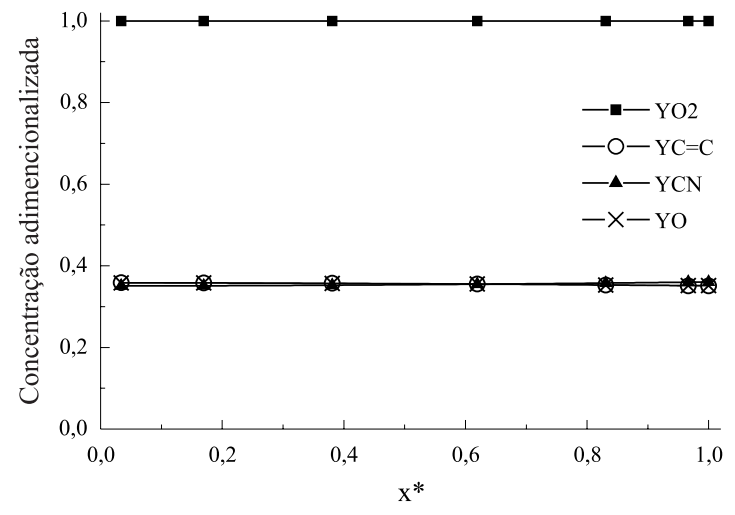

Figura 4. Perfis de concentrações na fita em função de $x^{*}$ depois de 2500 segundos de estabilização, para valores de $\mathrm{N}+1=7, \alpha=0$ e $\beta=0$.
Tabela 1. Condições de estabilização para o caso 1.

\begin{tabular}{lc}
\hline & Caso 1 \\
\hline Espessura da fita $(\mathrm{e}, \mathrm{cm})$ & 0,1 \\
Raio do filamento $\left(\mathrm{R}_{\mathrm{fil},}, \mathrm{cm}\right)$ & $5,5 \mathrm{e}-4$ \\
Número de filamentos & 50000 \\
Temperatura inicial do forno $\left(\mathrm{T}_{\mathrm{xi}},{ }^{\circ} \mathrm{C}\right)$ & 240 \\
Temperatura inicial da fita $\left(\mathrm{T}_{\mathrm{fi}},{ }^{\circ} \mathrm{C}\right)$ & 25 \\
Velocidade do ar do forno $(\mathrm{v}, \mathrm{cm} / \mathrm{s})$ & 300 \\
Perfil de temperatura do forno & Isotérmico \\
\hline
\end{tabular}

colocação, $\alpha=0$ e $\beta=0$ (as sub-rotinas e respostas para as raízes obtidas nestas condições podem ser encontradas em Villadsen (1970)). Devido à pequena variação das concentrações e temperaturas com a espessura da fita, analisaram-se os resultados para uma posição $x^{*}$ fixa de 0,3807 , em função do tempo de estabilização, estes resultados foram comparados com os obtidos para o feixe de fibras PAN (Rosa, 1999).

\section{Perfis de temperaturas e concentrações em função do tempo}

O modelo ao longo do tempo para um simples perfil isotérmico do forno é apresentado para discutir o gradiente de temperatura e composição na fita e comparar com os perfis obtidos para o modelo de feixe.

A temperatura, na posição $x^{*}$ de 0,3807 , de uma fita de 50000 filamentos em um forno a $240{ }^{\circ} \mathrm{C}$, é apresentado na Figura 5. As condições para este caso estão resumidas na Tabela 1 .

Como as reações são exotérmicas, a temperatura da fita fica acima da temperatura do forno. Porém, observa-se que no caso de uma fita (Figura 5), o acúmulo de calor é bem menor do que no feixe (Figura 6). $\mathrm{Na}$

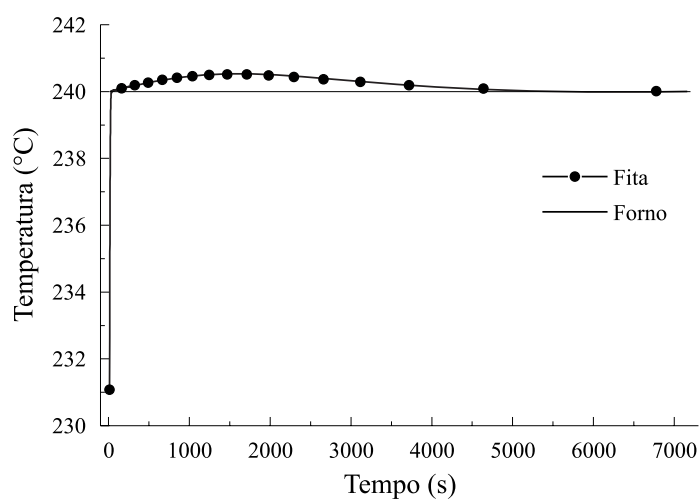

Figura 5. Perfil de temperatura durante a estabilização termo-oxidativa para uma fita de fibras PAN. 


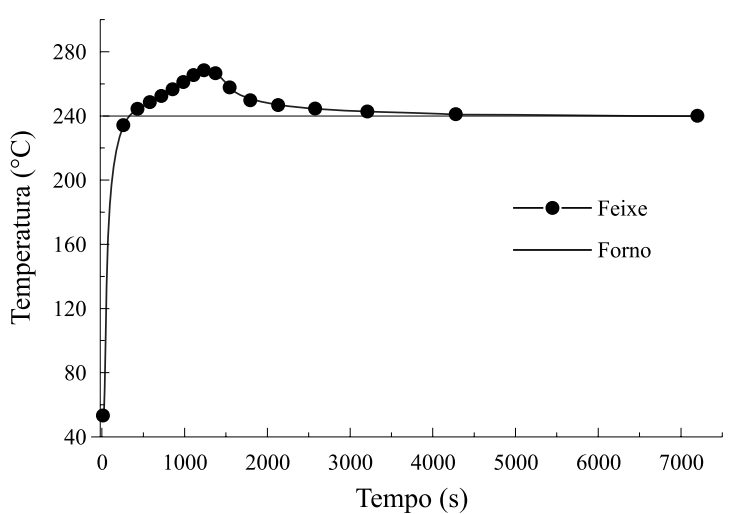

Figura 6. Perfil de temperatura durante a estabilização termo-oxidativa para um feixe de fibras PAN (Rosa, 1999).

fita a temperatura fica somente $1{ }^{\circ} \mathrm{C}$ acima da temperatura do forno, enquanto no feixe este aumento é de aproximadamente $40{ }^{\circ} \mathrm{C}$.

No modelo de feixe foram consideradas as mesmas condições da Tabela 1, com um raio do feixe de 0,1625 representado, na Figura 6.

A estabilização em um feixe para as condições do caso 1 apresentou um comportamento meta-estável para a temperatura (Figura 6), o que não é indicado para a estabilização, pois nestas condições as reações exotérmicas podem ficar descontroladas, causando cisão de cadeia ou até queima do feixe. Neste caso seria importante melhorar as condições de transferência de calor ou diminuir a temperatura do forno. Para a estabilização de uma fita nas condições do caso 1 (Figura 5) observou-se um perfil mais estável com as reações exotérmicas mais controladas. Este comportamento pode ser explicado justamente pela opção da disposição plana das fibras que, pelas leis de Fick, garantem uma melhor difusão de oxigênio como também uma melhor de transferência de calor. Desta forma, a maioria dos processos comerciais de estabilização utilizam a disposição plana das fibras

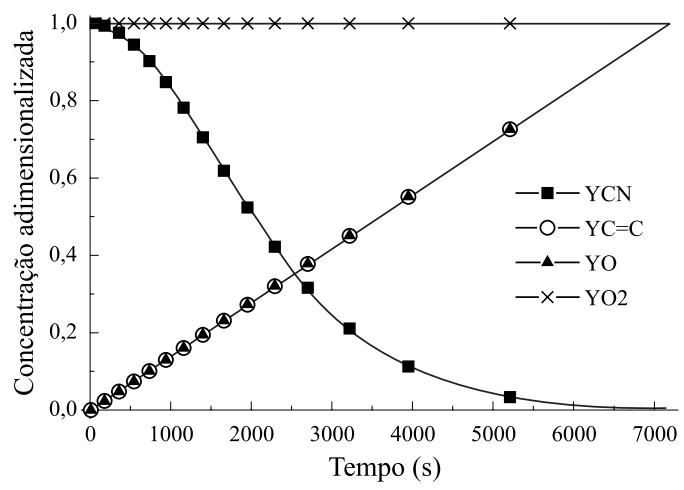

Figura 7. Perfis de concentrações durante a estabilização termo-oxidativa para uma fita de fibras PAN.

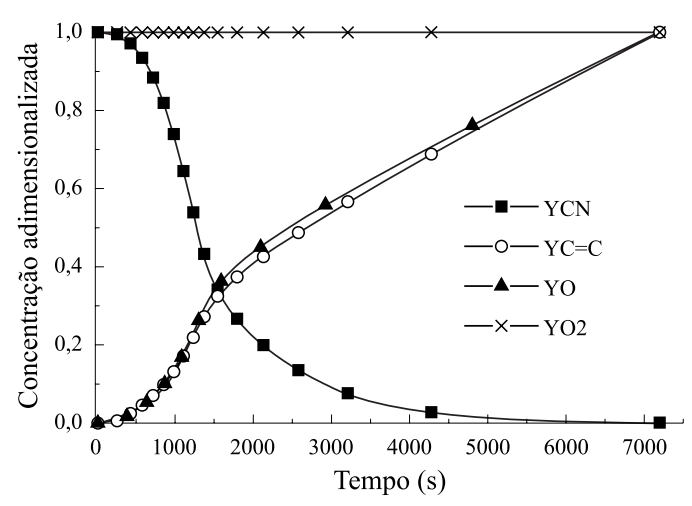

Figura 8. Perfis de concentrações durante a estabilização termo-oxidativa para um feixe de fibras PAN (Rosa, 1999).

(fita), de modo a garantir uma melhor e controlada reação.

Nas Figuras 7 e 8 verifica-se o comportamento das espécies reagindo com o tempo, para fita e feixe. Como esperado os grupos nitrila são consumidos e as ligações oxigênio e duplas ligações são formadas com o tempo. A difusão de oxigênio é rápida e não causa diferença na concentração de gás oxigênio com o tempo.

Os perfis obtidos no feixe indicam que as reações são mais rápidas do que no caso da fita e isto se deve ao maior aumento da temperatura no feixe. Estes perfis mostram que a estabilização na fita é muito mais controlada do que em feixe.

Os estudos realizados em feixes (Rosa, 1999) ilustram a importância da temperatura no processo de estabilização e mostram que a velocidade do fluxo de ar no feixe e o tamanho do feixe são ferramentas importantes para controlar as reações exotérmicas de estabilização. No entanto, em fitas estes parâmetros não interferem tanto, pois a transferência de calor na fita é melhor e portanto as reações exotérmicas mais controladas. Um conjunto de simulações foi realizado com a intenção acompanhar o efeito da temperatura e número de filamentos no feixe e na fita de fibras PAN.

\section{Estudo da temperatura isotérmica do forno}

A Tabela 2 apresenta as condições utilizadas para ilustrar o efeito da temperatura isotérmica do forno na fita de fibras PAN. Para um feixe de fibras PAN de raio 0,1625 foram considerados os casos 1,2 e 3 com as temperaturas de 240,220 e $200{ }^{\circ} \mathrm{C}$ e as demais condições são as mesmas utilizadas para a fita.

Para uma melhor visualização do efeito da temperatura isotérmica do forno agruparam-se os perfis de temperaturas e concentrações para os casos 1, 2, 3 e 4 . 
Tabela 2. Condições utilizadas nas simulações para os casos 2, 3 e 4 com um aumento da temperatura do forno.

\begin{tabular}{lccc}
\hline & Caso 2 & Caso 3 & Caso 4 \\
\hline $\mathrm{e}(\mathrm{cm})$ & 0,1 & 0,1 & 0,1 \\
$\mathrm{R}_{\mathrm{fil}}(\mathrm{cm})$ & $5,5 \mathrm{e}-4$ & $5,5 \mathrm{e}-4$ & $5,5 \mathrm{e}-4$ \\
$\mathrm{n}$. fil. & 50000 & 50000 & 50000 \\
$\mathrm{~T}_{\mathrm{xi}}\left({ }^{\circ} \mathrm{C}\right)$ & 220 & 260 & 300 \\
$\mathrm{~T}_{\mathrm{fi}}\left({ }^{\circ} \mathrm{C}\right)$ & 25 & 25 & 25 \\
$\mathrm{v}(\mathrm{cm} / \mathrm{s})$ & 300 & 300 & 300 \\
Perfil (forno) & Isotérmico & Isotérmico & Isotérmico \\
\hline
\end{tabular}

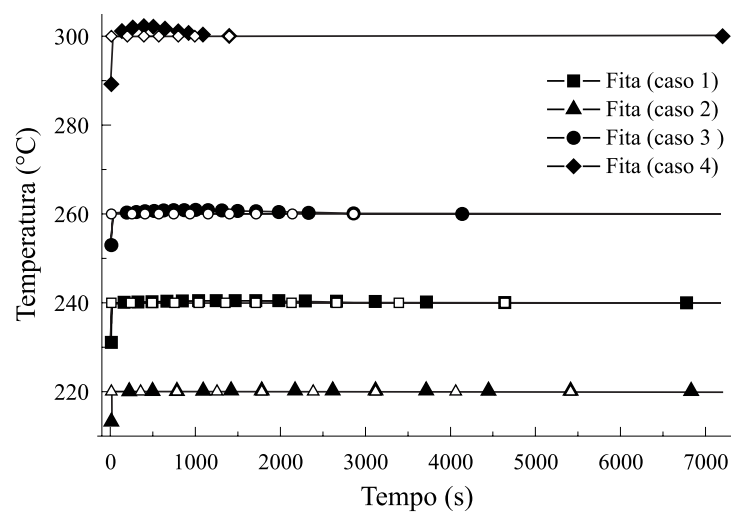

Figura 9. Análise comparativa dos perfis de temperaturas durante a estabilização para as condições do caso 1, 2, 3 e 4 na fita de fibras PAN.

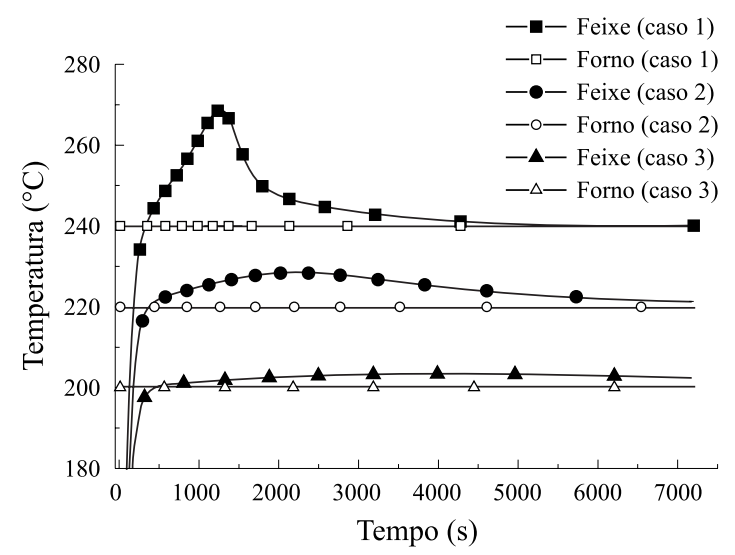

Figura 10. Análise comparativa dos perfis de temperaturas durante a estabilização para as condições do caso 1, 2 e 3 no feixe de fibras PAN.

$\mathrm{Na}$ Figura 9 apresentam-se os perfis de temperatura para os casos 1, 2, 3 e 4 na fita de fibras e na Figura 10 os perfis de temperatura para os casos 1,2 e 3 no feixe de fibras.

Na Figura 11 apresentam-se os perfis de concentrações para os casos 1, 2, 3 e 4 na fita de fibras e na Figura 12 os perfis de temperatura para os casos 1, 2 e 3 no feixe de fibras.

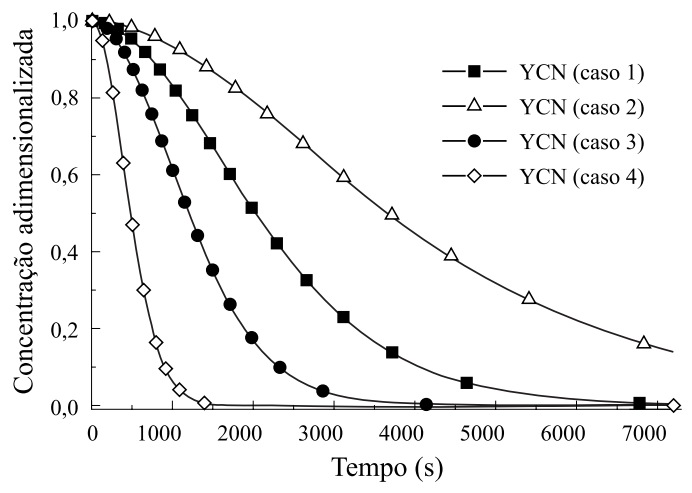

Figura 11. Análise comparativa dos perfis de concentrações de grupos nitrila durante a estabilização para a fita nas condições do caso 1, 2,3 e 4 .

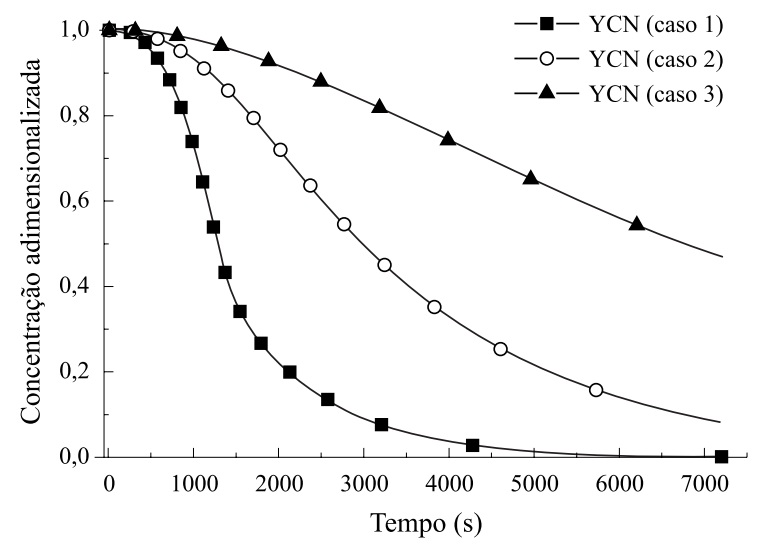

Figura 12. Análise comparativa dos perfis de concentrações de grupos nitrila durante a estabilização para o feixe nas condições do caso 1,2 e 3 .

Estudo do número de filamentos em uma fita e em um feixe de fibras PAN

Na Tabela 3 estão as condições utilizadas para a estabilização da fita de fibras PAN. Os perfis de temperaturas e concentrações para esta simulação estão apresentados nas Figuras 13 e 15. Para um feixe foram considerados os casos 7, 8 e 9 com 30000,10000

Tabela 3. Condições para as simulações para os casos 7 e 8 com variações no número de filamentos da fita.

\begin{tabular}{lcc}
\hline & Caso 7 & Caso 8 \\
\hline $\mathrm{e}(\mathrm{cm})$ & 0,06 & 0,02 \\
$\mathrm{R}_{\mathrm{fil}}(\mathrm{cm})$ & $5,5 \mathrm{e}-4$ & $5,5 \mathrm{e}-4$ \\
$\mathrm{n}$. fil. & 30000 & 10000 \\
$\mathrm{~T}_{\mathrm{xi}}\left({ }^{\circ} \mathrm{C}\right)$ & 240 & 240 \\
$\mathrm{~T}_{\mathrm{fi}}\left({ }^{\circ} \mathrm{C}\right)$ & 25 & 25 \\
$\mathrm{v}(\mathrm{cm} / \mathrm{s})$ & 300 & 300 \\
Perfil do forno & Isotérmico & Isotérmico \\
\hline
\end{tabular}




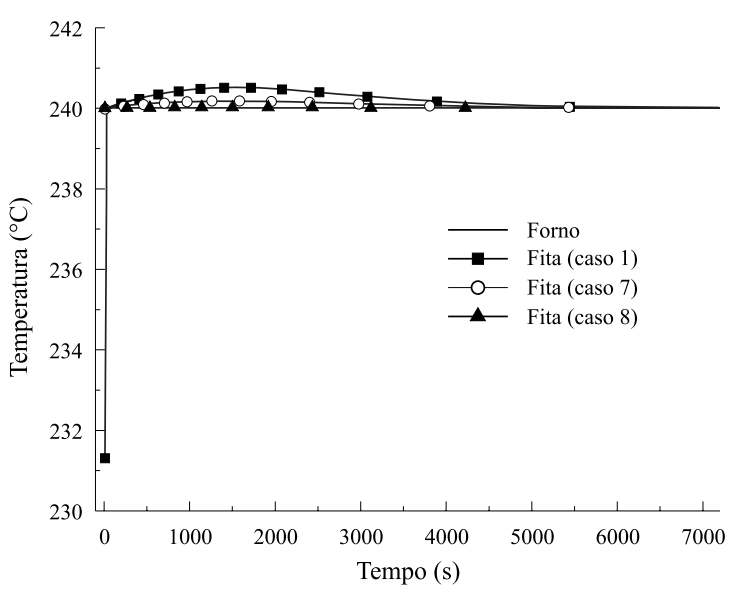

Figura 13. Análise comparativa dos perfis de temperaturas durante a estabilização para as condições do caso 1,7 e 8 na fita de fibras PAN.

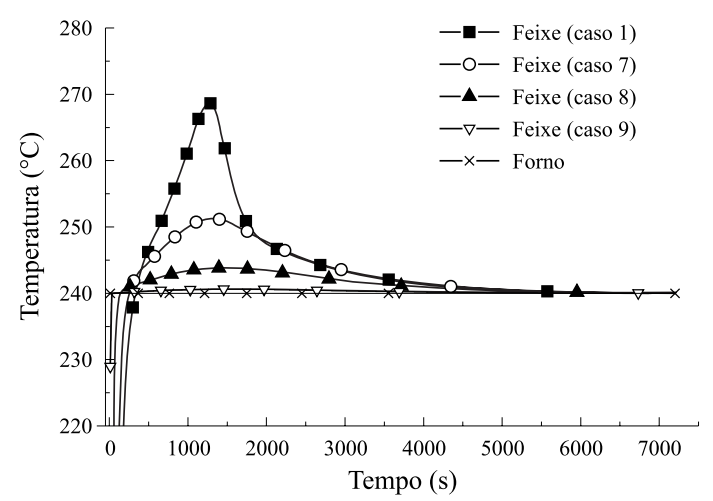

Figura 14. Análise comparativa dos perfis de temperaturas durante a estabilização para as condições $1,7,8$ e 9 no feixe de fibras PAN.

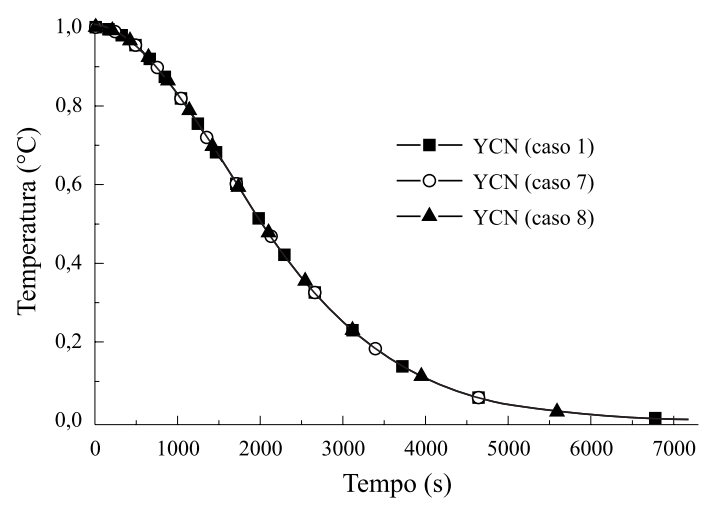

Figura 15. Análise comparativa dos perfis de concentrações de grupos nitrila durante a estabilização para a fita nas condições do caso 1,7 e 8 .

e 1000 filamentos e raios de 0,1259, 0,0726 e 0,0229 respectivamente (Figuras 14 e 16).

Os casos apresentados indicam que as alterações na temperatura do forno e no número de filamentos na fita não causam grandes mudanças nos perfis de temperatura e concentrações na fita de fibras. Desta forma, pode-se afirmar que é muito mais difícil con-

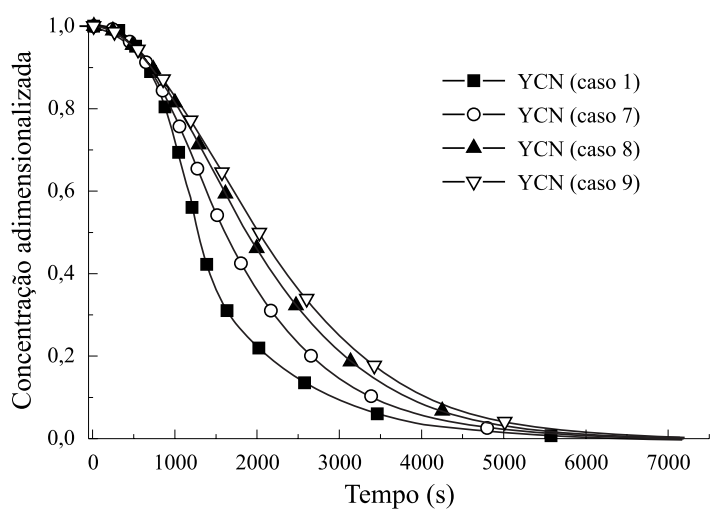

Figura 16. Análise comparativa dos perfis de concentrações de grupos nitrila durante a estabilização para o feixe nas condições do caso $1,7,8$ e 9 .

trolar as reações exotérmicas em um feixe do que em uma fita de fibras PAN.

\section{Conclusões}

Este trabalho mostrou que o processo de estabilização termo-oxidativa pode ser modelado para fitas de fibras PAN e os métodos matemáticos utilizados apresentaram-se adequados para a resolução das equações do modelo.

Comparando-se os perfis de temperaturas em função do tempo para fitas e feixes de fibras PAN observou-se um perfil muito mais estável e com as reações exotérmicas mais controladas na fita do que no feixe, já que a disposição plana das fibras leva a uma melhor transferência de calor. Desta forma, a estabilização de fitas de fibras garante um maior controle das reações químicas exotérmicas garantindo a qualidade do produto.

O estudo através da modelagem matemática da estabilização de fitas de fibras PAN, pode contribuir para o avanço de uma tecnologia nacional de obtenção de fibras estabilizadas oxidativamente (fibras PANOX). Trata-se de um estudo que vem sendo desenvolvido junto ao Centro Técnico Aeroespacial e possíveis testes serão realizados para confirmação do modelo.

\section{Agradecimentos}

Agradecemos à FAPESP pela concessão de bolsa de estudos, processo: 00/03007-6.

\section{Referências Bibliográficas}

1. Rosa, A. M. A. - Dissertação de Mestrado, UNICAMP, (1999). 
2. Del'Arco, A. P. J. - Tese de Doutorado, UNICAMP, (1997).

3. Finlayson, B. A. - Nonlinear Analysis in Chemical Engineering, New York, McGraw-Hill, Inc., (1980).

4. Grove III, D. A.; Abhiraman, A. S. - Carbon, 30, 45 (1992).

5. Collins, G. L.; Thomas, N. W.; Williams, G. E. - Carbon 26, 671 (1988).

6. Villadsen, J. E.; Michelsen, M. L. - Solution of Differential Equation Models by Polinimial Aproximation, New Jersey, Prentice-Hall, Inc. (1978).
7. Villadsen, J. - Selected Aproximation Methods For Chemical Engineering Problems, Lyngby, Danmarks Tekniske Hojshole (1970).

8. Dunham, M. G.; Edie, D. D. - Carbon, 30, 435 (1992).

9. Michelsen, M. L. - An Efficient General Purpose Method For The Integration Of Stiff Ordinary Differential Equations, A. I. Ch. E. Journal, 22(3): 5947 (1976).

Recebido: 10/08/01

Aprovado: 11/06/02 